\title{
Probing in cell protein structural changes with time-resolved X-ray scattering $\dagger$
}

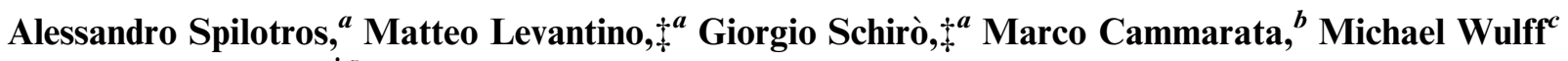 \\ and Antonio Cupane $* a$
}

Received 16th February 2012, Accepted 23rd April 2012

DOI: $10.1039 / \mathrm{c} 2 \mathrm{sm} 25676 \mathrm{~b}$

\begin{abstract}
Investigating protein structural changes inside the cell is a major goal in molecular biology. Here we show that time-resolved wideangle X-ray scattering is a valuable tool for this purpose. Hemoglobin has been chosen as a model system and its tertiary and quaternary conformational changes following laser flash-photolysis have been tracked in intact red blood cells with nanosecond time resolution.
\end{abstract}

Proteins are molecular machines whose structure is finely tailored to perform their biological tasks. Regulation of protein functioning often requires structural changes occurring over a wide range in time (from sub-picoseconds to seconds) and space (from subangstroms to tens of angstroms) scales. Modeling biological systems and investigating the structural, dynamic and functional behavior of proteins in cells are nowadays considered one of the most important challenges in life sciences. ${ }^{1,2}$ Experimental data from model systems and structural characterizations vs. protein concentration suggest that molecular crowding and geometrical confinement, which characterize the intracellular environment, may influence protein structure and dynamics. ${ }^{3}$ Protein structural changes and their effect on the equilibrium atomic fluctuations can be inferred by analyzing the properties of the equilibrium structures. These kinds of studies have been also performed on cellular systems by means of nuclear magnetic resonance ${ }^{4-6}$ and neutron scattering. ${ }^{7-10}$ Nevertheless, it is evident that time-resolved techniques able to track nonequilibrium protein structural changes occurring in cells in response to a chemical or light induced excitation in real time are needed. Moreover, given the heterogeneity of the intracellular environment, a technique able to single out the protein structural signal, while avoiding cell disruption, is required. Timeresolved optical spectroscopy studies have been used to monitor ligand binding kinetics in cells, ${ }^{11}$ however they give signals only indirectly related to protein three-dimensional structures. Nuclear

${ }^{a}$ University of Palermo, Department of Physics, Via Archirafi 36, I-90123 Palermo, Italy. E-mail: antonio.cupane@unipa.it

${ }^{b}$ Centre National de la Recherche Scientifique, Institut de Physique de Rennes, 35042 Rennes, France

${ }^{c}$ European Synchrotron Radiation Facility, 38043 Grenoble, France

$\dagger$ Electronic supplementary information (ESI) available. See DOI: $10.1039 / \mathrm{c} 2 \mathrm{sm} 25676 \mathrm{~b}$

\$ These authors contributed equally to the work. magnetic resonance, although sensitive to the structure of proteins in the cell, ${ }^{4-6}$ works best for small and properly labeled proteins and it has millisecond time resolution. ${ }^{12}$ Time-resolved wide-angle X-ray scattering (TR-WAXS) using synchrotron radiation has the potentiality to yield significant information on protein structural changes inside the cell since it is a technique directly sensitive to protein structure and has up to 100 ps time resolution. TR-WAXS has been successfully applied to investigate the conformational changes of proteins in solution after photoexcitation with nanosecond ${ }^{13-15}$ and picosecond ${ }^{16,17}$ laser pulses. In particular, the tertiary and quaternary conformational changes involved in the R-T allosteric transition of human hemoglobin $(\mathrm{Hb})$, a paradigm of cooperativity in molecular biology, have been tracked and their time scale assessed. ${ }^{13,15}$

The aim of this work is to demonstrate the applicability of TRWAXS to track protein structural changes directly in the cellular environment by investigating the time course of the $\mathrm{R}-\mathrm{T}$ allosteric transition of $\mathrm{Hb}$ inside intact red blood cells (RBCs) following laser flash-photolysis. The challenge associated with such experiments is to single out the $\mathrm{Hb}$ time dependent scattering signals from those of other cell components. From this point of view, adult RBCs have the advantage of being rich in $\mathrm{Hb}\left(\sim 330 \mathrm{mg} \mathrm{m}^{-1}\right)$ and of lacking the nucleus and most of the organelles.

In TR-WAXS experiments on carbon monoxide hemoglobin ( $\mathrm{HbCO})$ in aqueous solution, a laser pulse $(\lambda=527 \mathrm{~nm}, \Delta t=250 \mathrm{~ns})$ triggered the sequence of protein conformational changes by photolyzing the bond between the $\mathrm{CO}$ ligands and $\mathrm{Hb}$, and the transient structures were probed by delayed X-ray pulses. ${ }^{13,15}$ Structural changes occurring at different time delays left their "fingerprints" in the differences between the scattering patterns measured before (laser off) and after (laser on) the photolysis pulse. The following experimental challenges arise when the same approach is applied to human RBCs suspended in an isotonic aqueous solution: (i) in RBC suspensions the local concentration may change with time due to precipitation thus producing spurious time-dependent changes in the scattering signal; (ii) because of visible light scattering from $\mathrm{RBC}$ and light absorption from $\mathrm{Hb}$, the penetration depth of the photolyzing laser beam is limited to tens of microns; (iii) X-rays are scattered not only from $\mathrm{Hb}$ but also from other molecules in the sample (including membranes, membrane proteins, etc.); (iv) X-ray absorption may cause the formation of free radicals ${ }^{18}$ as a result of water radiolysis and affect membrane integrity. ${ }^{19}$ To overcome 
the above mentioned experimental challenges, we performed the experiment on a densely packed layer of RBCs, thus avoiding RBC precipitation during the measurement and minimizing visible light scattering. ${ }^{11}$ Nevertheless, under these conditions the laser penetration depth is limited to $\sim 100 \mu \mathrm{m}$ due to the high $\mathrm{Hb}$ concentration ( $\sim 4.4 \mathrm{mM}$ in tetramer). In order to deliver the X-ray beam within $100 \mu \mathrm{m}$ from the sample holder surface, we developed a new mylar sample holder that avoids parasitic signals from the quartz surface of standard X-ray capillaries. Laser on - laser off difference signals were used to subtract scattering contributions from sample components other than $\mathrm{Hb}$. Solvent heating contributions induced by the energy transferred from the laser into the solvent via protein absorption were removed by subtracting, from the difference signal at each time delay, the $32 \mathrm{~ms}$ difference signal (when $\mathrm{Hb}$ recovers its equilibrium $\mathrm{HbCO}$ structure, but the energy deposited by the laser pulse has not yet diffused out of the probed volume). ${ }^{13}$ The experimental setup, the "laser on" - "laser off" subtraction, the heating signal, and the effectiveness of the mylar cell are shown in Fig. 1 and Fig. $2 \mathrm{a}$ and $\mathrm{b}$ respectively. The rather high phosphate buffer concentration $(\sim 130 \mathrm{mM})$ minimized the damaging effect of free radicals while ensuring nearly isotonic conditions ( $290 \mathrm{mOsm}){ }^{20}$ As is commonly done in X-ray scattering experiments, a translation stage was used to "dilute" the potential laser/X-ray damage over a large sample volume. Our approach ensured that essentially no RBC lysis occurred during data acquisition (Fig. 2c-e); microscope images acquired after several hours of irradiation show modest effects on the RBC volume and membrane integrity. The scattered intensity below $0.2 \AA^{-1}$ (not shown in Fig. 1b), which is sensitive to the intermolecular $\mathrm{Hb}$ distance, ${ }^{21}$ was monitored during the data acquisition in order to check the sample integrity in real time (see ESI $\dagger$ ). All experiments have been performed on beamline ID9B at the European Synchrotron Radiation Facility in Grenoble (France). ${ }^{22}$

Fig. 3a shows a comparison between the shape of normalized difference WAXS data from packed RBCs and from a $1 \mathrm{mM} \mathrm{Hb}$ aqueous solution at two relevant time delays that, as already shown by Cammarata et al. for $\mathrm{Hb}$ solutions, ${ }^{13,15}$ are representative of the tertiary ( $316 \mathrm{~ns}$, top panel) and quaternary (31.6 $\mu \mathrm{s}$, bottom panel) relaxations, respectively. The close similarity in the shape of the data reported in Fig. 3a demonstrates that: (i) our procedure is effective in isolating the protein structural signal and (ii) we are able to detect tertiary and quaternary conformational changes of $\mathrm{Hb}$ inside RBCs. TR-WAXS difference patterns, $\Delta S$, measured at several time delays on packed RBCs are compared in Fig. 3b with analogous data relative to a $1 \mathrm{mM} \mathrm{Hb}$ aqueous solution. Although the time evolution of the two datasets is clearly different, it cannot be straightforwardly concluded that the $\mathrm{R}-\mathrm{T}$ allosteric transition in the two samples occurs either on different time scales or in the same time scale. Indeed, the high $\mathrm{Hb}$ concentration in packed RBCs is such that, right after photolysis, the unbound $\mathrm{CO}$ concentration is sizably higher than for the $\mathrm{Hb}$ solution; this explains the large difference between the two samples in the time scale of bimolecular CO rebinding and thus in the time evolution of the TR-WAXS signals. Moreover, the photolysis pulse generates a heterogeneous distribution of $\mathrm{Hb}$ molecules sharing the same quaternary structure but differing in the number of bound ligands; since each species undergoes the $\mathrm{R}-\mathrm{T}$ allosteric transition with a rate that depends on the number of bound ligands, it is clear that differences in the

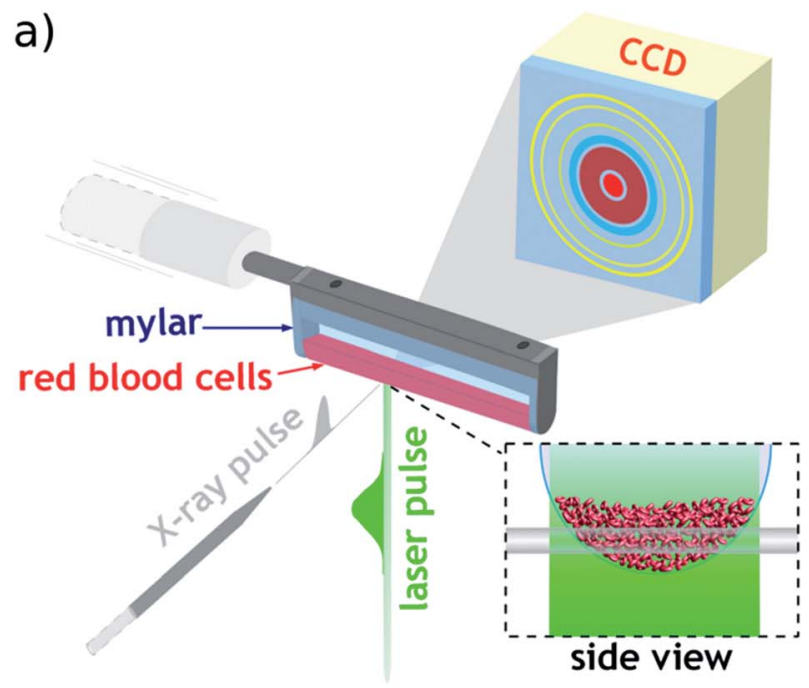

b)

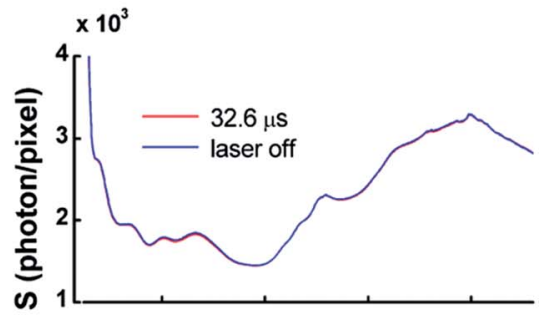

C)

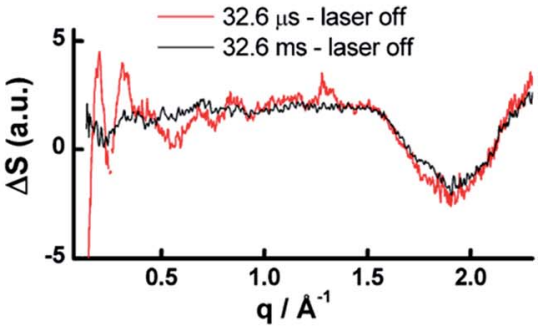

Fig. 1 TR-WAXS set-up and data processing: (a) schematic representation of the experimental setup: a laser pulse (green beam) photolyzes the Hb$\mathrm{CO}$ bond and triggers the sequence of protein conformational changes whose time course is monitored by delayed quasi-monochromatic X-ray pulses (100 ps long) extracted from the synchrotron; scattered X-rays are recorded in the forward direction by an X-ray sensitive CCD camera. All experiments have been performed at $20^{\circ} \mathrm{C}$ on $\sim 100 \mu \mathrm{l}$ of packed RBCs. (b) One-dimensional $S(q)$ curves have been obtained from azimuthal averaging of CCD images plus conversion from the scattering angle $\theta$ to the scattering vector magnitude $q=4 \pi \operatorname{sen}(\theta / 2) / \lambda$, where $\lambda=0.6793 \AA$ is the X-ray peak wavelength. (c) After normalization, the $S(q)$ pattern obtained from the unexcited sample ("laser off" pattern) is subtracted from the scattering pattern at a given time delay ("laser on" pattern); these "laser on - laser off" difference patterns, $\Delta S(q)$, are "fingerprints" of transient protein structural changes and also contain contributions related to the solvent heating induced by the energy absorbed from the photolysis laser (the maximum temperature jump, estimated from the heating signal, is $\sim 6^{\circ} \mathrm{C}$ ); the latter contribution can been removed from the data by subtraction of the 32 ms difference pattern. ${ }^{13,15}$ 


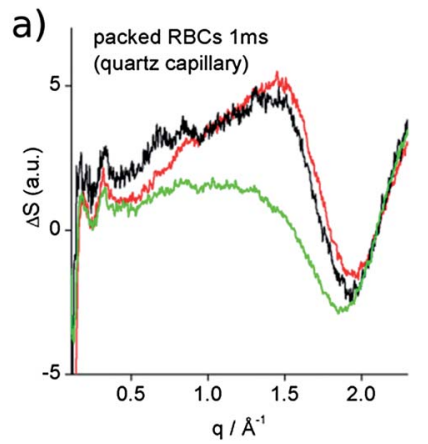

c)

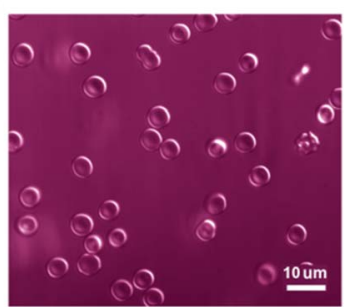

d)

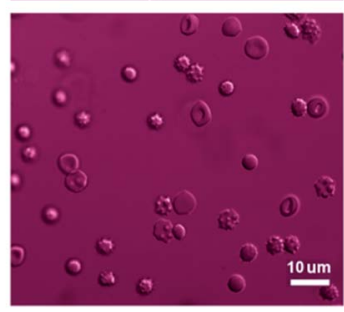

b) packed RBCs $1 \mathrm{~ms}$

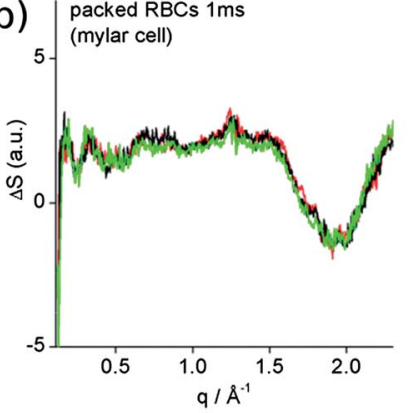

e)

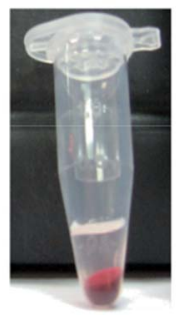

a)
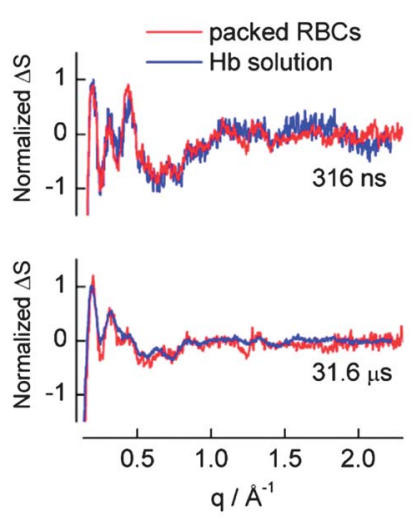

b)

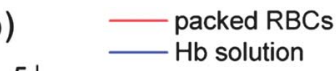

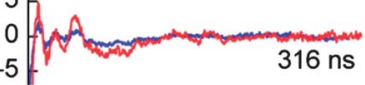
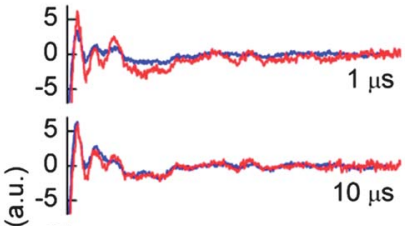

(i) $^{-5}$

$\infty$

5
-5

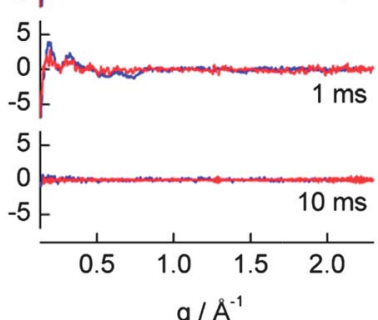

Fig. 3 Comparison of packed RBCs with a Hb aqueous solution: (a) normalized TR-WAXS difference patterns at $316 \mathrm{~ns}$ (top panel) and 31.6 $\mu$ s (bottom panel) of packed RBCs (red curves) and of a $1 \mathrm{mM} \mathrm{Hb}$ solution (blue curves); the curves have been normalized to their maximum at $0.19 \AA^{-1}$ to facilitate shape comparison; the solvent response has been removed from all patterns by subtraction of the $32 \mathrm{~ms}$ difference pattern. At both time delays, the difference patterns have essentially the same shape demonstrating the capability of TR-WAXS to track tertiary and quaternary conformational changes occurring inside the cell. (b) TRWAXS patterns (solvent response subtracted) at selected time delays from photolysis of packed RBCs (red curves) and of a $1 \mathrm{mM} \mathrm{Hb}$ solution (blue curves); the time evolution of the two datasets is clearly different (it is not possible to scale one dataset onto the other with a single scaling factor). in view of the high transmittance of mylar to X-rays, a much smaller background signal is obtained thus minimizing the above effect. (c and d) Microscope images of the RBC sample before (panel c) and after (panel d) a prolonged X-ray exposure; note that the two samples have been diluted in the same proportion before taking the images. (e) Picture of the RBC sample collected after X-ray exposure and centrifuged in order to check globule integrity: the supernatant solution is colorless indicating that essentially all $\mathrm{Hb}$ molecules are still confined inside the RBCs.

fraction of photolyzed $\mathrm{Hb}$ subunits also contribute to the observed signal time evolution. In order to get quantitative information on the kinetics of conformational changes, it is necessary to analyze the whole TR-WAXS dataset obtained with packed RBCs in terms of a model able to take into account the above mentioned heterogeneity of the processes occurring after $\mathrm{HbCO}$ photolysis. Here we have used the Monod-Wyman-Changeux (MWC) kinetic model, already successfully applied to the analysis of TR-WAXS data on $\mathrm{Hb}$ solutions. ${ }^{15,23}$ The scheme of the kinetic model is depicted in Fig. 4a, representative fittings of RBC patterns are reported in Fig. $4 \mathrm{~b}$, and the best fit parameters are given in Table 1. Further details on data analysis are reported in the $\mathrm{ESI}^{22}$ and in previous publications. ${ }^{13-15}$ Our analysis shows that the time evolution of RBC data can be reproduced with high fidelity using the same kinetic parameters as those obtained from previous experiments on $\mathrm{Hb}$ in

aqueous solution once the total $\mathrm{CO}$ concentration and the fraction of photolyzed $\mathrm{Hb}$ subunits are properly taken into account. ${ }^{22} \mathrm{We}$ conclude that: (i) the structural intermediates along the $\mathrm{R}-\mathrm{T}$ kinetic pathway are the same whether $\mathrm{Hb}$ is inside the $\mathrm{RBC}$ or in a dilute solution (see Fig. 3a); (ii) our data are compatible with an essentially unaltered time scale of the R-T allosteric transition (Fig. $4 \mathrm{~b}$ and Table 1).

\section{Conclusions}

The TR-WAXS study presented here is the first application of this technique to a cell system and we succeeded in tracking the tertiary and quaternary conformational changes of a protein in intact cells. The results suggest that the crowding and confinement experienced inside RBCs do not affect appreciably the functionally relevant R-T quaternary relaxation of human $\mathrm{Hb}$ as well as the involved transient structures. We anticipate that the availability of different kinds of excitations (e.g. laser induced $\mathrm{pH}$ jumps or temperature jumps) and/ or the possibility of overexpressing proteins in bacteria will allow to extend in cell TR-WAXS studies to different and more complicated biological processes, such as protein folding-unfolding or light transduction by transmembrane proteins. 


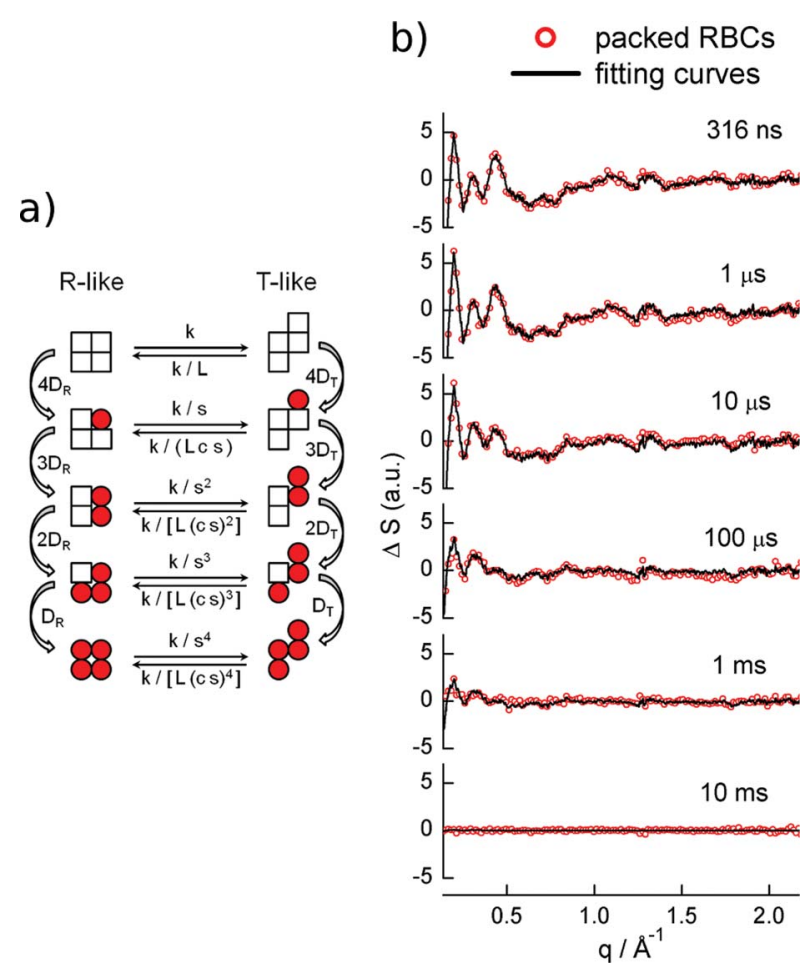

Fig. 4 Results of TR-WAXS data analysis: (a) scheme of the MWC allosteric kinetic model used to fit the data: each protein can be in either the $\mathrm{R}$ or the $\mathrm{T}$ quaternary state resulting in ten different possible microscopic states $\mathrm{R}_{i}$ and $\mathrm{T}_{i}$ (where $i=0 \ldots 4$ is the number of bound $\mathrm{CO}$ molecules per $\mathrm{Hb}$ molecule); interconversion between microscopic states can occur through the allosteric R-T transition or bimolecular ligand rebinding. $k$ is the rate of the $\mathrm{R}_{0}-\mathrm{T}_{0}$ transition, $s$ is a factor that takes into account the slowing down of the $\mathrm{R}-\mathrm{T}$ transition with increasing number of bound ligands, $L$ is the $\left[\mathrm{T}_{0}\right] /\left[\mathrm{R}_{0}\right]$ ratio, $c$ is the $K_{\mathrm{T}} / K_{\mathrm{R}}$ ratio (where $K_{\mathrm{T}}$ and $K_{\mathrm{R}}$ are the equilibrium association constants to $\mathrm{T}$ and $\mathrm{R}$ species, respectively), and $D_{\mathrm{T}}$ and $D_{\mathrm{R}}$ are the bimolecular rebinding rates to $\mathrm{T}$ and $\mathrm{R}$ species, respectively. (b) Comparison between the data relative to packed RBCs reported in Fig. 3b (red symbols) and the relative fitting curves obtained from the model (black lines). Errors on experimental data are of the order of the symbols size.

\section{Acknowledgements}

This work was supported by the Italian MIUR grant PRIN 2008 to A.C. We thank L. Eybert for help in the realization of the mylar
Table 1 Kinetic parameters of the model ${ }^{a}$

\begin{tabular}{lll}
\hline & Hb in packed $\mathrm{RBCs}^{b}$ & $\mathrm{Hb}^{b}$ in solution \\
\hline$[\mathrm{Hb}](\mathrm{mM})$ & 4.4 & 1 \\
$N_{0}$ & $0.52 \pm 0.01$ & $0.78 \pm 0.01$ \\
$1 / k=\tau\left(\mathrm{R}_{0}-\mathrm{T}_{0}\right)(\mu \mathrm{s})$ & $1.2 \pm 0.1$ & $1.2 \pm 0.1$ \\
$s$ & 13 & $13 \pm 2$ \\
$L$ & 8850 & 8850 \\
$c$ & $3.3 \times 10^{-3}$ & $(3.3 \pm 0.2) \times 10^{-3}$ \\
$D_{\mathrm{R}}\left(\mu \mathrm{M}^{-1} \mathrm{~s}^{-1}\right)$ & 7.9 & 7.9 \\
$D_{\mathrm{T}}\left(\mu \mathrm{M}^{-1} \mathrm{~s}^{-1}\right)$ & 0.031 & 0.031
\end{tabular}

${ }^{a}$ Errors on best fit parameters represent the range of variability during the fitting procedure; parameters without errors have been kept fixed. ${ }^{b}$ Buffer: $130 \mathrm{mM}$ phosphate at $\mathrm{pH}$ 7.4. ${ }^{c}$ Buffer: $100 \mathrm{mM}$ phosphate at $\mathrm{pH} 7.0$.

sample holder and D. Skoufias for kindly providing access to the microscope lab at IBS in Grenoble.

\section{Notes and references}

1 B. Di Ventura, C. Lemerle, K. Michalodimitrakis and L. Serrano, Nature, 2006, 443, 527.

2 A. S. Smith, Nat. Phys., 2010, 6, 726.

3 H. X. Zhou, G. Rivas and A. P. Minton, Annu. Rev. Biophys., 2008, 37, 375 .

4 Z. Serber, et al., J. Am. Chem. Soc., 2001, 123, 2446.

5 D. Sakakibara, et al., Nature, 2009, 458, 102.

6 K. Inomata, et al., Nature, 2009, 458, 106.

7 W. Doster and S. Longeville, Biophys. J., 2007, 93, 1360.

8 J. Pieper, A. Buchsteiner, N. A. Dencher, R. E. Lechner and T. Hauss, Photochem. Photobiol., 2009, 85, 590.

9 A. M. Stadler, et al., Biophys. J., 2008, 95, 5449.

10 A. M. Stadler, et al., J. R. Soc. Interface, 2011, 8, 590.

11 L. J. Parkhurst and Q. H. Gibson, J. Biol. Chem., 1967, 242, 5762.

12 P. Schanda, V. Forge and B. Brutscher, Proc. Natl. Acad. Sci. U. S. A., 2007, 104, 11257.

13 M. Cammarata, et al., Nat. Methods, 2008, 5, 881.

14 M. Andersson, et al., Structure, 2009, 17, 1265.

15 M. Cammarata, M. Levantino, M. Wulff and A. Cupane, J. Mol. Biol., 2010, 400, 951.

16 H. S. Cho, et al., Proc. Natl. Acad. Sci. U. S. A., 2010, 107, 7281.

17 K. H. Kim, et al., Chem. Commun., 2011, 47, 289.

18 M. Weik, et al., Proc. Natl. Acad. Sci. U. S. A., 2000, 97, 623.

19 S. Bashir, F. Naik, R. Cardigan and S. Thomas, Vox Sanguinis, 2011, 101, 200.

20 E. B. Hendry, Clin. Chem., 1961, 7, 156.

21 S. Krueger and R. Nossal, Biophys. J., 1988, 53, 97.

22 ESI $\uparrow$.

23 J.-P. Changeux, Annu. Rev. Biophys., 2011, 41, 83. 